\title{
Treatment of Multiple Myeloma in Elderly Patients: A Review of Literature and Practice Guidelines
}

\author{
Suresh Manapuram ${ }^{1}$, Hamza Hashmi ${ }^{2}$ \\ 1. Internal Medicine, Saint Francis Hospital and Medical Center, Grand Island, USA 2. Oncology, University of \\ Louisville School of Medicine, Louisville, USA
}

Corresponding author: Hamza Hashmi, hamzahashmi87@hotmail.com

\begin{abstract}
Multiple myeloma (MM) is a clonal disorder of malignant plasma cells that comprises approximately $10 \%$ of hematologic malignancies. With median age of 66 at the time of presentation, multiple myeloma is predominantly a disease of the elderly. The availability of new combination regimens and the enhanced safety of autologous hematopoietic stem cell transplant has increased the treatment options for elderly patients with multiple myeloma. We provide a summary of data supporting the current management of elderly patients with newly diagnosed multiple myeloma.
\end{abstract}

Categories: Oncology

Keywords: multiple myeloma, elderly, stem cell transplant

\section{Introduction And Background}

Multiple myeloma (MM) is a clonal disorder of malignant plasma cells that comprises approximately $10 \%$ of hematologic malignancies. The median age at diagnosis is 66 years, making it predominantly a disease of the elderly. Multiple myeloma accounts for approximately $1 \%-2 \%$ of all cancers and slightly more than $17 \%$ of hematologic malignancies in the United States [1]. The annual incidence of MM in the United States is approximately four to five per 100,000. The incidence increases with age and combined with the worldwide increase in the elderly population, there is an anticipated $77 \%$ increase in the number of patients older than 65 years diagnosed with MM each year by 2030 [2-4]. Development of newer therapeutic agents and improving supportive care over the last two decades has significantly improved the outcome of MM in younger patients [5-7]. However, most studies suggest that improvements are marginal in elderly patients (defined as age 75 years and older) [2, 6]. This may be explained by the higher incidence of more severe disease in the older patients, but it is mainly due to patient characteristics (e.g. performance status, comorbidities) and organ dysfunction associated with aging [5-7].

Received 11/06/2018 Review began 11/22/2018 Review ended 11/29/2018 Published 12/01/2018

\section{() Copyright 2018}

Manapuram et al. This is an open access article distributed under the terms of the Creative Commons Attribution License CC-BY 3.0., which permits unrestricted use, distribution, and reproduction in any medium, provided the original author and source are credited.
Patients older than age 75 are at increased risk of frailty, vulnerable to adverse events associated with highdose chemotherapy and they are rarely candidates for high dose therapy (HDT) plus autologous stem cell transplant (ASCT). However, the availability of new front-line treatment regimens based on the novel agents thalidomide, bortezomib, and lenalidomide have dramatically changed the management of MM in transplant-ineligible patients. In addition, the goals of therapy in the elderly patients may differ from younger patients; older adults are more likely to prioritize symptom control, prolonged treatment-free intervals, and good quality of life over prolonged survival $[2,8,9]$. In this review, we provide a summary of data supporting the current management of elderly patients with newly diagnosed multiple myeloma.

\section{Review}

\section{Therapy for non-transplant eligible patients}

The alkylating agent plus corticosteroid combination with melphalan and prednisone (MP) was the standard of care for over 30 years. The addition of novel agents to MP has provided several therapeutic options for ASCT-ineligible patients with MM.

\section{Thalidomide-based regimens}

The first novel agent to be combined with MP was thalidomide. Six randomized trials compared MP with MP plus thalidomide (MPT) as primary treatment in elderly patients [10-15]. The response rates and depth of response were significantly increased in the MPT arm in all six studies (overall response rate of 59\% for MPT vs. $37 \%$ for MP; $\mathrm{P}<0.001$ ) [16, 17]. The partial response (PR) rate was $42 \%-76 \%$ vs. $28 \%-48 \%$ with MPT and MP, respectively, and progression-free survival (PFS) was 14-28 versus 10-19 months. Overall survival (OS) was significantly improved in the MPT arm in three out of six studies. A meta-analysis of the pooled data from the six MPT trials showed that addition of thalidomide to MP as a front-line regimen in elderly MM patients is associated with a significant improvement in PFS (5.4 months of benefit; hazard ratio (HR) of 
0.67 ; confidence interval (CI) of 0.55-0.80) and a near significant improvement in OS (6.6 months of benefit; HR of 0.82; CI of 0.66-1.02) [16]. Based on these studies, the combination of MP with thalidomide improves response rates and PFS, but with increased toxicity.

The combination of thalidomide and dexamethasone (TD) was compared with MP in a randomized study in elderly MM patients. While the response rate, including complete response (CR) rate, was superior in the TD arm, there was no benefit in terms of PFS and OS and was significantly shorter in the TD arm, particularly in patients over 75 years of age $[18,19]$. These results were explained by higher toxicities and early mortality.

Another randomized study compared the efficacy and safety of cyclophosphamide, thalidomide and dexamethasone (CTD) with MP in elderly patients [20]. CTD improved response rates compared with MP; however, PFS and OS rates were similar with the two regimens. CTD was also associated with higher rates of thromboembolic complications and infections.

\section{Lenalidomide-based regimens}

Lenalidomide, thalidomide's next generation analog, is more potent and less toxic than thalidomide. A randomized phase III trial compared MP versus a combination of melphalan, prednisone, and lenalidomide with or without lenalidomide maintenance (MPR or MPR-R) [21]. Preliminary results of the study showed MPR-R was significantly superior to MP with higher response rates (77\% vs. $50 \%)$ and greater CR rates (18\% vs. 5\%), superior to MPR in terms of PFS [2, 21-23]. The toxicity profile is less when compared with MPT and the common adverse events observed were neutropenia, thrombocytopenia, and infections.

A randomized Eastern Cooperative Oncology Group (ECOG) study compared lenalidomide and high-dose dexamethasone (40 mg for four days on, four days off, Revlimid dexamethasone [high dose] (RD)) with lenalidomide and low-dose dexamethasone (40 mg once per week, Revilmid dexamethasone [low dose] (Rd)) in newly diagnosed MM patients [24]. The Rd group was superior to RD with a median PFS of 25.3 months and $87 \%$ two-year OS. The RD regimen was associated with higher overall response rate (ORR) than $\mathrm{Rd}$; however, this improvement in response came at the expense of increased toxicity. This trial has been the basis for current use of low dose dexamethasone in almost all patients with MM.

In the Frontline Investigation of Lenalidomide plus Dexamethasone versus Standard Thalidomide (FIRST) trial involving 1623 newly diagnosed MM patients who were transplant ineligible, patients were randomly assigned to MPT for 12 cycles (approximately 17 months), Rd for the same fixed period, and Rd continuously [25]. This trial showed a significant increase in PFS with continuous Rd compared with MPT or Rd for 18 cycles (25.5 months for continuous Rd, 20.7 months for 18 cycles of Rd, and 21.2 months for MPT). Fouryear OS was significantly superior for continuous Rd over MPT [5, 25].

\section{Bortezomib-based regimens}

Bortezomib, the first-in-class proteasome inhibitor, is another important option for frontline therapy in elderly patients with MM. A large phase III randomized trial called Velcade as Initial Standard Therapy in Multiple Myeloma (VISTA) compared MP and bortezomib plus MP (MPV) in a series of 682 newly diagnosed MM patients [26]. MPV was significantly superior to MP in ORR ( $71 \%$ vs. $35 \%$ ), CR rates (30\% vs. $4 \%$ ). An updated analysis of the data with a median follow-up of five years demonstrated significant OS benefit with MPV vs. MP, with 13.3 month increase in the median OS (56.4 months vs. 43.1 months) [27]. Based on these data, MPV is recognized as standard of care regimen for use in elderly patients. However, safety was a concern in elderly patients; in the MPV arm, the incidence of grade 3 or grade 4 peripheral neuropathy was $14 \%$, and $30 \%$ of patients had to discontinue treatment or at least discontinue bortezomib because of treatment-related adverse events [26].

A reduction in bortezomib toxicity with equal efficacy was demonstrated for weekly (rather than twice weekly) once dosing in the following trials [28-30]. In a Spanish study with bortezomib, melphalan, and prednisone (VMP) induction with reduced intensity bortezomib followed by maintenance therapy with bortezomib-thalidomide (VT) or bortezomib-prednisone (VP), the results showed that after six cycles, the incidence of grade 3 or 4 neuropathy dropped to $7 \%$ with an ORR of $80 \%$ (20\% of CR) [5, 30]. In another study, VMP induction (nine cycles) without maintenance was compared with VMP plus thalidomide (VMPT) followed by maintenance therapy with VT [22, 31]. In both the arms, weekly bortezomib was associated with significantly decreased frequency of severe neuropathy. VMPT-VT improved response rates and PFS [31].

\section{Carfilzomib-based combinations}

Carfilzomib, a second-generation proteasome inhibitor, emerged as a new therapeutic option for newly diagnosed transplant-ineligible MM patients. In a pilot phase I/II study, carfilzomib was combined with MP (KMP) and the results are promising with good response rates and an acceptable toxicity profile. Randomized trials comparing KMP with VMP are currently running and the results are awaited [32]. In another study, a combination of carfilzomib with cyclophosphamide and low dose dexamethasone (KCydex) has been evaluated in a series of 53 newly diagnosed elderly MM patients, resulting in an ORR rate of $91 \%$ and a CR rate of $47 \%$. The regimen was safe and well-tolerated with no grade 3 or 4 neuropathy reported [33]. 


\section{Current role of HDT plus ASCT in elderly patients}

The great majority of randomized controlled trials comparing HDT plus ASCT and conventional-dose chemotherapy have excluded adults older than age 65 years [34]. However, ASCT is feasible in patients older than 65 years, at least in selected patients with good performance status and no severe comorbidities. Over the past two decades, the proportion of adults older than 70 years undergoing ASCT increased more than five-fold, from less than $1 \%$ to $5 \%$ [2, 35]. Lower doses of melphalan $(100-140 \mathrm{mg} / \mathrm{m} 2)$ are used for patients older than 70 years. Mehta et al. recommended avoiding ASCT in elderly patients with significantly compromised renal function unless it is clearly related to active myeloma that is unresponsive to other therapy [22]. Certain treatment-related toxicities are more common in the older adults, when compared with younger patients, and commonly include cardiac arrhythmias ( $8 \%$ vs. $0 \%$ ) and GI toxicity ( $68 \%$ vs. $46 \%$ grade 3-4 diarrhea; $45 \%$ vs. $23 \%$ grade $3-4$ oral toxicity) [36, 37]. Comorbidities are associated with greater toxicity and increased length of hospital stay.

\section{Effectiveness of HDT plus ASCT in elderly patients}

In the absence of randomized studies on the efficacy of ASCT in elderly patients with MM, several cohort studies have compared the response rates, PFS and OS with ASCT between older and younger patients. An Italian study has proposed two to three courses of melphalan, $100 \mathrm{mg} / \mathrm{m} 2$, supported by ASCT [38] and demonstrated that this approach was superior to conventional chemotherapy with MP in patients aged 50 to 70 years, including patients aged 65 to 70 years (44 in 4). Another trial, the Intergroupe Francophone du Myelome (IFM) 99-06, reported conflicting results regarding the benefit of ASCT over conventional therapy in older adults. In the IFM 99-06 trial, patients aged 65-75 years were randomly assigned to MP alone, MPT, or induction chemotherapy followed by two courses of melphalan $(100 \mathrm{mg} / \mathrm{m} 2)$ with ASCT [11]. In this trial, the MPT regimen yielded a higher response and CR rate than MP and was significantly superior both to MP and to melphalan plus ASCT.

In a post hoc analysis of a phase II study, the subgroups of patients older than age 65 years who underwent ASCT were compared with those who did not [39]. Both groups received thalidomide-based induction and transplant ineligible patients received two additional cycles of chemotherapy followed by thalidomide maintenance. The ASCT group had higher response rates; however, there was no benefit in PFS and OS when compared to non ASCT group.

The use of novel agents in combination with intermediate-dose melphalan might improve results by increasing the efficacy/toxicity ratio of induction treatment and by delaying relapses with post ASCT consolidation/maintenance. An Italian group studied the combination regimen of bortezomib combined with doxorubicin and dexamethasone (PAD regimen) in a group of 102 patients aged 65 to 75 years (45 in 4). The induction treatment was effective with the rate of CR plus very good partial response (VGPR) $58 \%$. In this study, $90 \%$ of patients received the first ASCT and $83 \%$ received the second ASCT. Patients received lenalidomide consolidation/maintenance therapy after ASCT, and the final rate of CR plus VGPR increased to $78 \%$ with two-year PFS and OS rates of $69 \%$ and $86 \%$, respectively.

\section{Challenges in treating elderly multiple myeloma patients}

Deciding how aggressively to treat MM in the elderly is a clinical challenge that needs a thorough understanding of indications and possible outcomes of the treatment. The decision to initiate combination chemotherapy should be taken by a multidisciplinary team considering all available diagnostic and clinical information and in discussion with the patient and their family. Comprehensive geriatric assessment (CGA) tools have been created to clinically assess frailty, functional status, comorbidities, cognitive ability, and nutritional status [40-42]. A specific CGA has been developed for assessment of MM patients to help guide treatment decisions in elderly patients [43]. This score was developed by a pooled analysis of data from three clinical trials consisting of 869 patients $[33,43]$. The discussion of treatment should include all alternatives, including palliative and complementary approaches. Given the increased rates of disease-related and treatment-related complications in the elderly patients, providing appropriate supportive care is of vital importance.

\section{Special considerations}

When considering treatment options for MM in the older adults, certain age-related comorbidities may play a vital role in choosing one regimen over the other. Thalidomide and bortezomib can be used at full doses in the presence of renal dysfunction and with hemodialysis, whereas the dose of lenalidomide needs adjustment. Older people are at increased risk of venous thromboembolism (VTE) because of impaired mobility. Risk of VTE is further enhanced with the use of immunomodulatory agents, thalidomide and lenalidomide in elderly MM patients. Anemia could be exacerbated by the chemotherapy and should be adequately managed with judicious red blood cell transfusion and intravenous iron infusion. Peripheral neuropathy is a common adverse effect of many MM therapies, such as thalidomide and bortezomib. The problem of peripheral neuropathy could be worrisome in MM in patients with diabetes. Elderly patients receiving MM therapy that affects gastrointestinal motility, such as bortezomib, are more prone to develop severe mucositis and diarrhea. Polypharmacy is quite prevalent in the older population. Thus, careful review of the patient's current list of medications and attention to potential drug-drug interactions is required. 


\section{Conclusions}

In conclusion, the availability of new combination regimens and enhanced safety of ASCT has increased the treatment options for elderly patients with multiple myeloma. Combination therapies including novel agents have improved the response and survival rates in transplant-ineligible patients. MPT, MPV, and lenalidomide/dexamethasone represent new standards of care for transplant-ineligible MM patients. It is important to assess each patient's clinical situation and fitness for therapy to allow treatment to be provided at an appropriate intensity. Particular care must be taken to minimize toxicities, reducing doses if required, to allow continuation of treatment when appropriate. Treating myeloma in the very elderly is challenging, and clinicians should develop a personalized approach to optimize the treatment response while minimizing toxicity through careful geriatric assessment and compassionate care.

\section{Additional Information \\ Disclosures}

Conflicts of interest: In compliance with the ICMJE uniform disclosure form, all authors declare the following: Payment/services info: All authors have declared that no financial support was received from any organization for the submitted work. Financial relationships: All authors have declared that they have no financial relationships at present or within the previous three years with any organizations that might have an interest in the submitted work. Other relationships: All authors have declared that there are no other relationships or activities that could appear to have influenced the submitted work.

\section{References}

1. Rajkumar SV, Kumar S: Multiple myeloma diagnosis and treatment. Mayo Clinic Proc. 2016, 91:101-19. 10.1016/j.mayocp.2015.11.007

2. Wildes TM, Rosko A, Tuchman SA: Multiple myeloma in the older adult: better prospects, more challenges. J Clin Oncol. 2014, 32:2531-40. 10.1200/JCO.2014.55.1028

3. Turesson I, Velez R, Kristinsson SY, Landgren O: Patterns of multiple myeloma during the past 5 decades: stable incidence rates for all age groups in the population but rapidly changing age distribution in the clinic. Mayo Clin Proc. 2010, 85:225-30. 10.4065/mcp.2009.0426

4. Smith BD, Smith GL, Hurria A, Hortobagyi GN, Buchholz TA: Future of cancer incidence in the United States: burdens upon an aging, changing nation. J Clin Oncol. 2009, 27:2758-65. 10.1200/JCO.2008.20.8983

5. Mateos MV, San Miguel JF: How should we treat newly diagnosed multiple myeloma patients? . Hematology Am Soc Hematol Educ Program. 2013, 2013:488-95. 10.1182/asheducation-2013.1.488

6. Pulte D, Gondos A, Brenner H: Improvement in survival of older adults with multiple myeloma: results of an updated period analysis of SEER data. Oncologist. 2011, 16:1600-1603. 10.1634/theoncologist.2011-0229

7. Kumar SK, Rajkumar SV, Dispenzieri A, et al.: Improved survival in multiple myeloma and the impact of novel therapies. Blood. 2008, 111:2516-20. 10.1182/blood-2007-10-116129

8. Rose JH, O'Toole EE, Dawson NV, et al.: Perspectives, preferences, care practices, and outcomes among older and middle-aged patients with late-stage cancer. J Clin Oncol. 2004, 22:4907-17. 10.1200/JCO.2004.06.050

9. Fried TR, Bradley EH, Towle VR, Allore H: Understanding the treatment preferences of seriously ill patients . N Engl J Med. 2002, 346:1061-6. 10.1056/NEJMsa012528

10. Palumbo A, Bringhen S, Caravita T, et al.: Oral melphalan and prednisone chemotherapy plus thalidomide compared with melphalan and prednisone alone in elderly patients with multiple myeloma: randomised controlled trial. Lancet. 2006, 367:825-31. 10.1016/S0140-6736(06)68338-4

11. Facon T, Mary JY, Hulin C, et al.: Melphalan and prednisone plus thalidomide versus melphalan and prednisone alone or reduced-intensity autologous stem cell transplantation in elderly patients with multiple myeloma (IFM 99-06): a randomised trial. Lancet. 2007, 370:1209-18. 10.1016/S01406736(07)61537-2

12. Hulin C, Facon T, Rodon P, et al.: Efficacy of melphalan and prednisone plus thalidomide in patients older than 75 years with newly diagnosed multiple myeloma: IFM 01/01 trial. J Clin Oncol. 2009, 27:3664-70. 10.1200/JCO.2008.21.0948

13. Wijermans P, Schaafsma M, Termorshuizen F, et al.: Phase III study of the value of thalidomide added to melphalan plus prednisone in elderly patients with newly diagnosed multiple myeloma: the HOVON 49 Study. J Clin Oncol. 2010, 28:3160-6. 10.1200/JCO.2009.26.1610

14. Waage A, Gimsing P, Fayers P, et al.: Melphalan and prednisone plus thalidomide or placebo in elderly patients with multiple myeloma. Blood. 2010, 116:1405-12. 10.1182/blood-2009-08-237974

15. Beksac M, Haznedar R, Firatli-Tuglular T, et al.: Addition of thalidomide to oral melphalan/prednisone in patients with multiple myeloma not eligible for transplantation: results of a randomized trial from the Turkish Myeloma Study Group. Eur J Haematol. 2011, 86:16-22. 10.1111/j.1600-0609.2010.01524.x

16. Fayers PM, Palumbo A, Hulin C, et al.: Thalidomide for previously untreated elderly patients with multiple myeloma: meta-analysis of 1685 individual patient data from 6 randomized clinical trials. Blood. 2011, 118:1239-47. 10.1182/blood-2011-03-341669

17. Kapoor P, Rajkumar SV, Dispenzieri A, et al.: Melphalan and prednisone versus melphalan, prednisone and thalidomide for elderly and/or transplant ineligible patients with multiple myeloma: a meta-analysis. Leukemia. 2011, 25:689-96. 10.1038/leu.2010.313

18. Ludwig H, Hajek R, Tothova E, et al.: Thalidomide-dexamethasone compared with melphalan-prednisolone in elderly patients with multiple myeloma. Blood. 2009, 113:3435-42. 10.1182/blood-2008-07-169565

19. Harousseau JL: Multiple myeloma in the elderly: when to treat, when to go to transplant. Oncology (Williston Park). 2010, 24:992-8. 
20. Morgan GJ, Davies FE, Gregory WM, et al.: Cyclophosphamide, thalidomide, and dexamethasone (CTD) as initial therapy for patients with multiple myeloma unsuitable for autologous transplantation. Blood. 2011, 118:1231-8. 10.1182/blood-2011-02-338665

21. Palumbo A, Hajek R, Delforge M, et al.: Continuous lenalidomide treatment for newly diagnosed multiple myeloma. N Engl J Med. 2012, 366:1759-69. 10.1056/NEJMoa1112704

22. Mehta J, Cavo M, Singhal S: How I treat elderly patients with myeloma . Blood. 2010, 116:2215-23. 10.1182/blood-2009-10-163329

23. Kapoor P, Kumar S, Fonseca R, et al.: Impact of risk stratification on outcome among patients with multiple myeloma receiving initial therapy with lenalidomide and dexamethasone. Blood. 2009, 114:518-21. 10.1182/blood-2009-01-202010

24. Rajkumar SV, Jacobus S, Callander NS, et al.: Lenalidomide plus high-dose dexamethasone versus lenalidomide plus low-dose dexamethasone as initial therapy for newly diagnosed multiple myeloma: an open-label randomised controlled trial. Lancet Oncol. 2010, 11:29-37. 10.1016/S1470-2045(09)70284-0

25. Benboubker L, Dimopoulos MA, Dispenzieri A, et al.: Lenalidomide and dexamethasone in transplantineligible patients with myeloma. N Engl J Med. 2014, 371:906-17. 10.1056/NEJMoa1402551

26. San Miguel JF, Schlag R, Khuageva NK, et al.: Bortezomib plus melphalan and prednisone for initial treatment of multiple myeloma. N Engl J Med. 2008, 359:906-17. 10.1056/NEJMoa0801479

27. San Miguel JF, Schlag R, Khuageva NK, et al.: Persistent overall survival benefit and no increased risk of second malignancies with bortezomib-melphalan-prednisone versus melphalan-prednisone in patients with previously untreated multiple myeloma. J Clin Oncol. 2013, 31:448-55. 10.1200/JCO.2012.41.6180

28. Kumar S, Rajkumar SV: ASH 2009 meeting report-top 10 clinically oriented abstracts in multiple myeloma . Am J Hematol. 2010, 85:210-213. 10.1002/ajh.21637

29. Mateos MV, Richardson PG, Schlag R, et al.: Bortezomib plus melphalan and prednisone compared with melphalan and prednisone in previously untreated multiple myeloma: updated follow-up and impact of subsequent therapy in the phase III VISTA trial. J Clin Oncol. 2010, 28:2259-66. 10.1200/JCO.2009.26.0638

30. Mateos MV, Oriol A, Martinez-Lopez J, et al.: Bortezomib, melphalan, and prednisone versus bortezomib, thalidomide, and prednisone as induction therapy followed by maintenance treatment with bortezomib and thalidomide versus bortezomib and prednisone in elderly patients with untreated multiple myeloma: a randomised trial. Lancet Oncol. 2010, 11:934-41. 10.1016/S1470-2045(10)70187-X

31. Palumbo A, Bringhen S, Rossi D, et al.: Bortezomib-melphalan-prednisone-thalidomide followed by maintenance with bortezomib-thalidomide compared with bortezomib-melphalan-prednisone for initial treatment of multiple myeloma: a randomized controlled trial. J Clin Oncol. 2010, 28:5101-9. 10.1200/JCO.2010.29.8216

32. Moreau P, Kolb B, Attal M, et al.: Phase 1/2 study of carfilzomib plus melphalan and prednisone in patients aged over 65 years with newly diagnosed multiple myeloma. Blood. 2015, 125:3100-4. 10.1182/blood-201502-626168

33. Bringhen S, Petrucci MT, Larocca A, et al.: Carfilzomib, cyclophosphamide, and dexamethasone in patients with newly diagnosed multiple myeloma: a multicenter, phase 2 study. Blood. 2014, 124:63-9. 10.1182/blood-2014-03-563759

34. Harousseau JL, Moreau P: Autologous hematopoietic stem-cell transplantation for multiple myeloma . N Engl J Med. 2009, 360:2645-54. 10.1056/NEJMct0805626

35. McCarthy PL Jr., Hahn T, Hassebroek A, et al.: Trends in use of and survival after autologous hematopoietic cell transplantation in North America, 1995-2005: significant improvement in survival for lymphoma and myeloma during a period of increasing recipient age. Biol Blood Marrow Transplant. 2013, 19:1116-23. 10.1016/j.bbmt.2013.04.027

36. Muta T, Miyamoto T, Fujisaki T, et al.: Evaluation of the feasibility and efficacy of autologous stem cell transplantation in elderly patients with multiple myeloma. Intern Med. 2013, 52:63-70.

37. Jantunen E, Kuittinen T, Penttila K, Lehtonen P, Mahlamäki E, Nousiainen T: High-dose melphalan (200 $\mathrm{mg} / \mathrm{m} 2$ ) supported by autologous stem cell transplantation is safe and effective in elderly (or=65 years) myeloma patients: comparison with younger patients treated on the same protocol. Bone Marrow Transplant. 2006, 37:917-22. 10.1038/sj.bmt.1705360

38. Palumbo A, Bringhen S, Bruno B, et al.: Melphalan $200 \mathrm{mg} / \mathrm{m}(2)$ versus melphalan $100 \mathrm{mg} / \mathrm{m}(2)$ in newly diagnosed myeloma patients: a prospective, multicenter phase 3 study. Blood. 2010, 115:1873-9. 10.1182/blood-2009-09-241737

39. Offidani M, Leoni P, Corvatta L, et al.: ThaDD plus high dose therapy and autologous stem cell transplantation does not appear superior to ThaDD plus maintenance in elderly patients with de novo multiple myeloma. Eur J Haematol. 2010, 84:474-83. 10.1111/j.1600-0609.2010.01418.x

40. Willan J, Eyre TA, Sharpley F, Watson C, King AJ, Ramasamy K: Multiple myeloma in the very elderly patient: challenges and solutions. Clin Interv Aging. 2016, 11:423-35. 10.2147/CIA.S89465

41. Hamaker ME, Jonker JM, de Rooij SE, Vos AG, Smorenburg CH, van Munster BC: Frailty screening methods for predicting outcome of a comprehensive geriatric assessment in elderly patients with cancer: a systematic review. Lancet Oncol. 2012, 13:437-44. 10.1016/S1470-2045(12)70259-0

42. Tuchman SA, Shapiro GR, Ershler WB, et al.: Multiple myeloma in the very old: an IASIA conference report . J Natl Cancer Inst. 2014, 106:dju067. 10.1093/jnci/dju067

43. Palumbo A, Bringhen S, Mateos MV, et al.: Geriatric assessment predicts survival and toxicities in elderly myeloma patients: an International Myeloma Working Group report. Blood. 2015, 125:2068-74. 10.1182/blood-2014-12-615187 